\title{
TECHNOLOGICAL INNOVATIONS AND OPPORTUNITIES FOR ENTREPRENEURSHIP DEVELOPMENT IN GEORGIA
}

\author{
AMIRANI MAGLAKELIDZE \\ Researcher \\ The Institute for the Research of Economic and Social Problems of Globalization, European University, Georgia \\ PhD Student \\ Ivane Javakhishvili Tbilisi State University, Georgia \\ maglakelidze.amirani@gmail.com
}

Abstract. Given the modern global conjuncture, technological innovation and creative thinking play a major role in the process of implementing entrepreneurial activities. The rapid development of digital technologies and the emergence of diverse digital platforms have given impetus to the transformation of entrepreneurial behavior and the conduct of entrepreneurial activities from the real - to the virtual environment. In the modern digital world, there is no alternative to technological entrepreneurship. 5G network, blockchain, 3D printing and other technological innovations make business processes faster, more reliable and flexible. The generation and implementation of continuous innovative ideas in technology has led to the emergence and development of information technology, digital technology, digital platforms, on which the modern form of entrepreneurship - digital entrepreneurship is based.

One of the obstacles to the development of digital entrepreneurship in Georgia is the lack of proper access to digital technologies. At the same time, the population of Georgia has not yet developed the digital culture and skills that ensure the implementation of commercial activities in the virtual space. According to the National Statistics Office of Georgia in July 2019 in the country: $70.6 \%$ of households actively used the Internet, only $20.8 \%$ of them purchased goods and/or services through digital channels. In 2018 , only $3.2 \%$ of enterprises received an order through the website. These data indicate the low level of Internet use by the public and, consequently, enterprises for commercial purposes. This untapped segment, at the same time, creates a solid potential for further development of digital entrepreneurship. Especially today, when physical mobility is restricted due to the worldwide pandemic COVID-19.

Innovations and technologies are the main driving forces of modern entrepreneurship. Therefore, the emphasis of the Government of Georgia in this direction and the launch of appropriate economic policy instruments should positively support the transformation of the entrepreneurial environment and entrepreneurial behavior in the country. Given the current situation in Georgia, in order to identify and develop digital entrepreneurial opportunities, it would be appropriate to take the following measures: First, the state must ensure that more people have access to the Internet and digital technologies; Second, increase the scale of financial support programs for innovative and digital entrepreneurial initiatives, also, to help startups attract funding sources, to consult on opportunities to attract venture investments, to make it possible to implement a more technologically innovative idea; Third, primacy should be given to Georgian universities in the fields of entrepreneurship, innovation and technology, computer science; Fourth, the network of innovation centers should be expanded and similar educational spaces should be created to ensure the development of technical and entrepreneurial skills of the population (especially the rural population) in the field of innovation and technology.

KEYWORDS: TECHNOLOGICAL INNOVATION, ENTREPRENEURSHIP, DIGITAL ENTREPRENEURSHIP, DIGITAL TECHNOLOGY, DIGITAL PLATFORM.

For citation: Maglakelidze, A. (2020). Technological Innovations and Opportunities for Entrepreneurship Development in Georgia. Globalization and Business, 10. 264-269. (In Georgian) https://doi.org/10.35945/gb.2020.10.036 


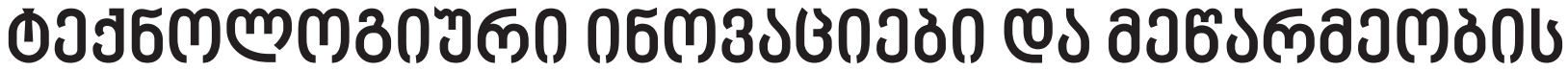

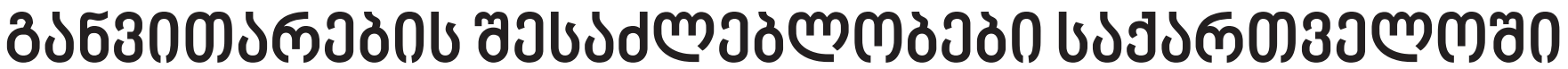

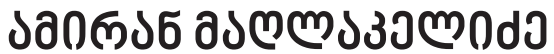

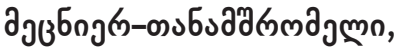

з мmmòumn comjom nुobon,

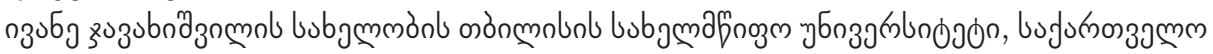

maglakelidze.amirani@gmail.com

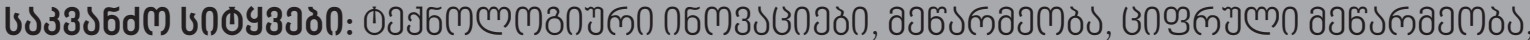

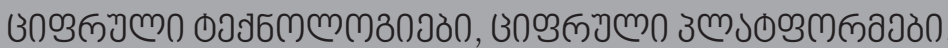

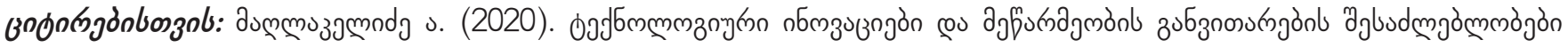

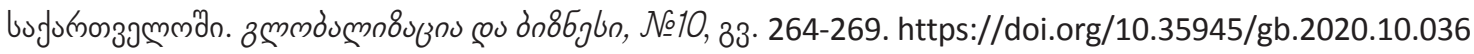

\section{əอเ১3১ल00}

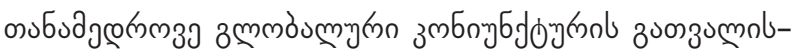

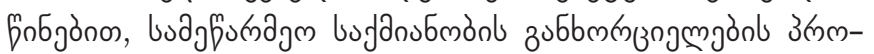

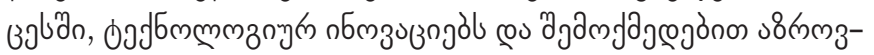

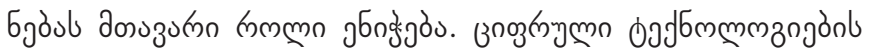

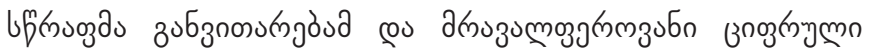

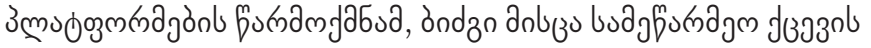

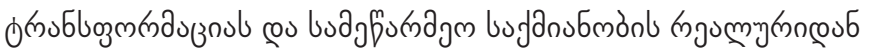

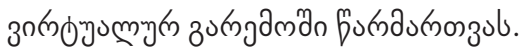

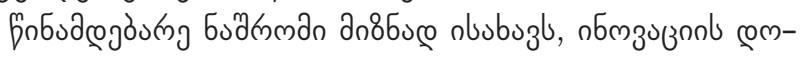

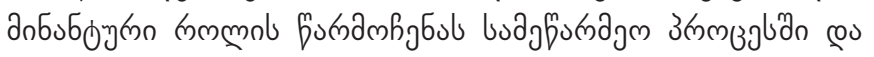

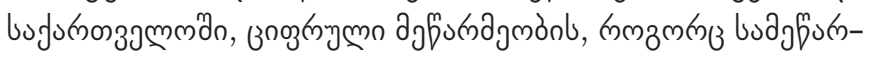

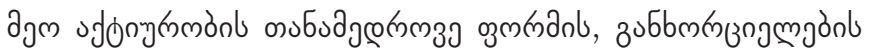

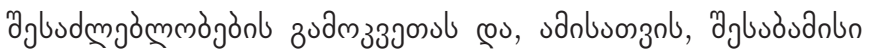

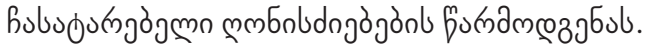

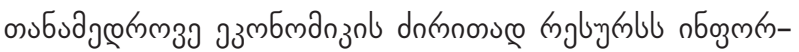

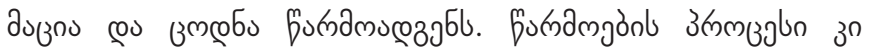

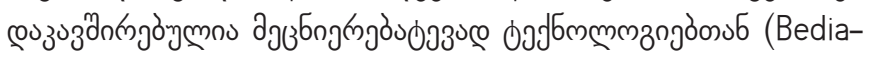

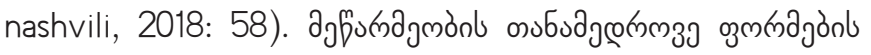

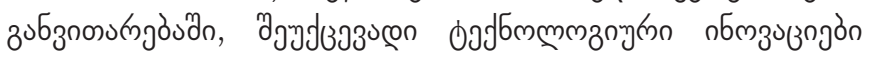

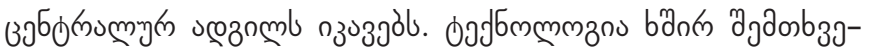

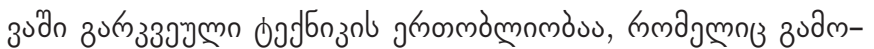

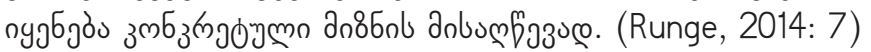

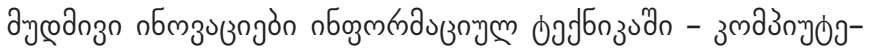

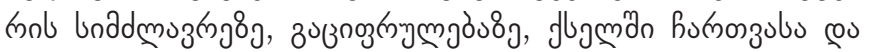

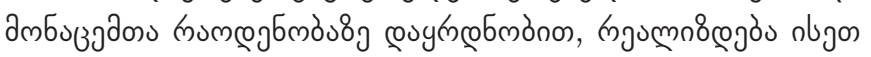

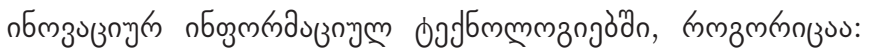

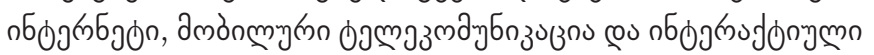

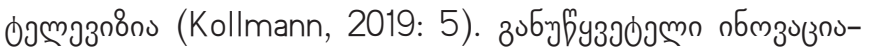

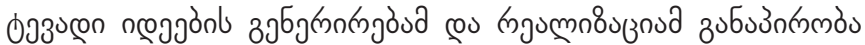

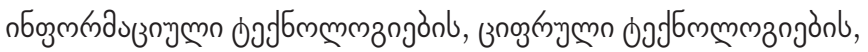

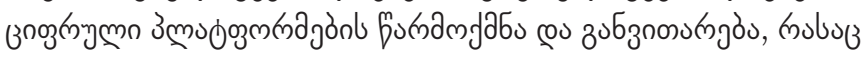

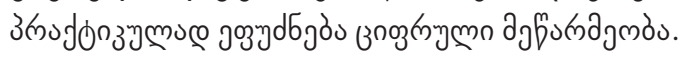

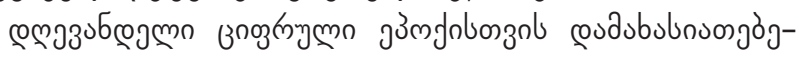

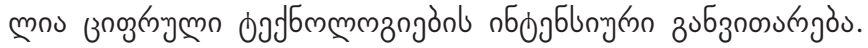

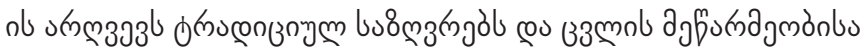

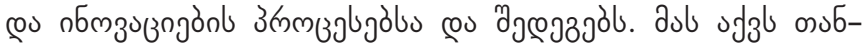

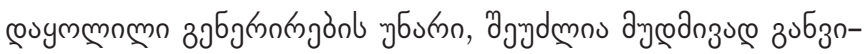

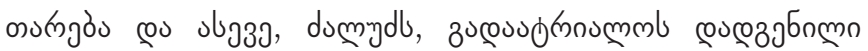

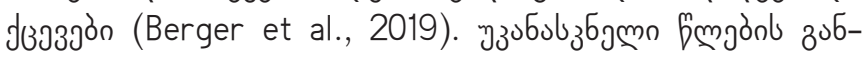

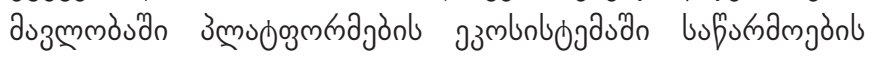

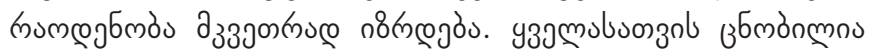

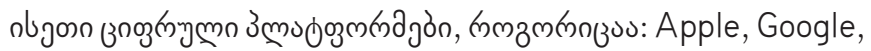

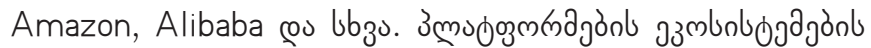

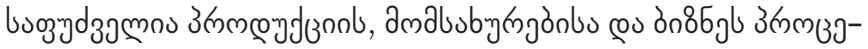

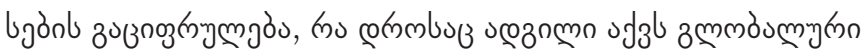
mubcoduogonl oुm nandngadul (Evans \& Gawer, 2016). am-

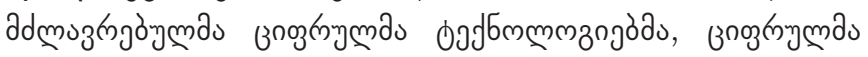

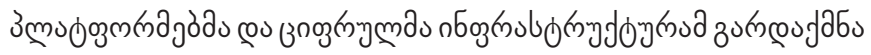

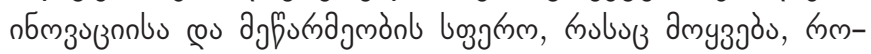

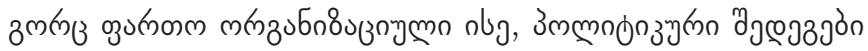
(Nambisan et al. 2019).

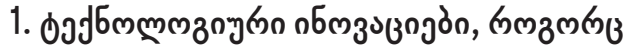

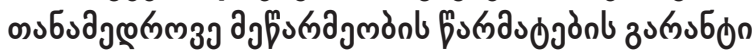

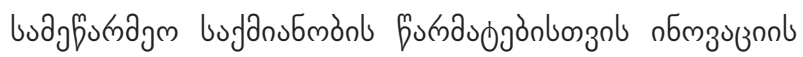

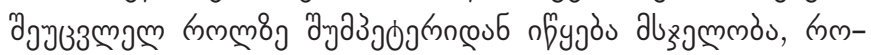

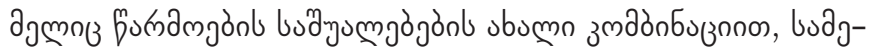




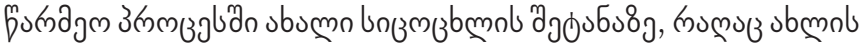

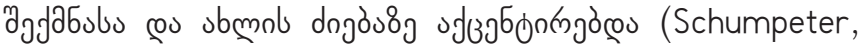

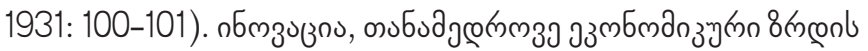

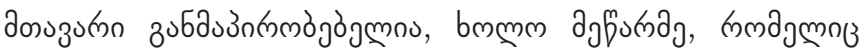

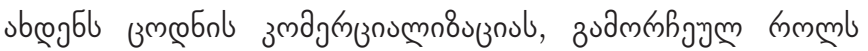

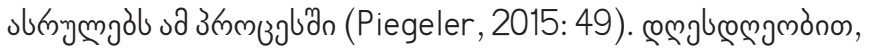

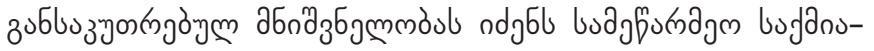

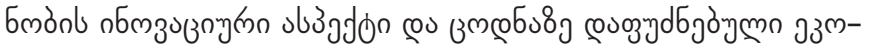

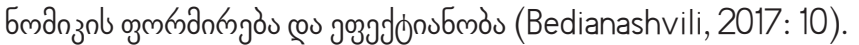

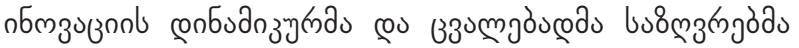

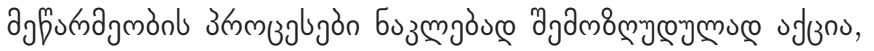

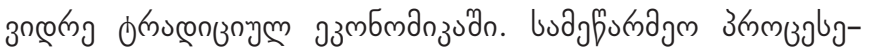

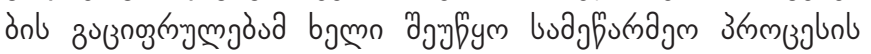

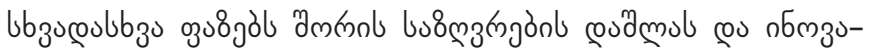

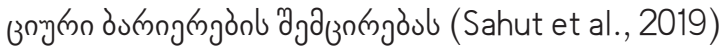

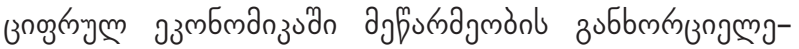

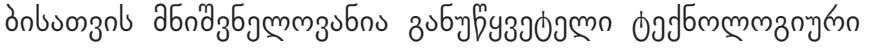

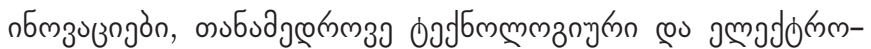

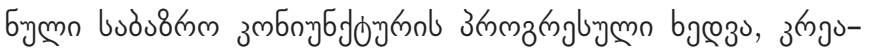

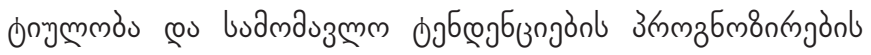

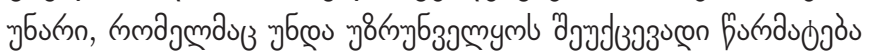
(Maglakelidze, 2020).

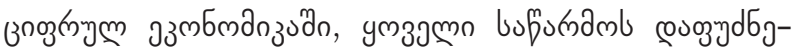

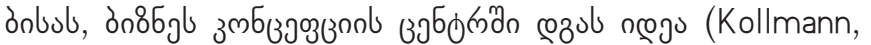

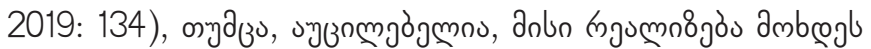

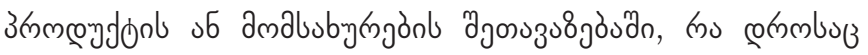
пzп ا ا

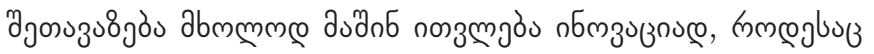

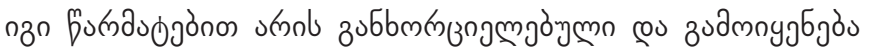

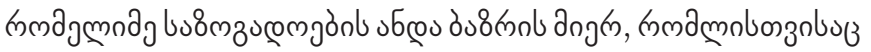

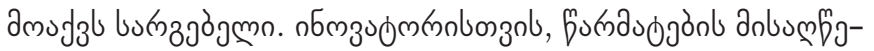

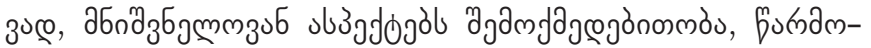

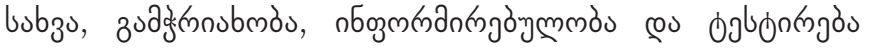

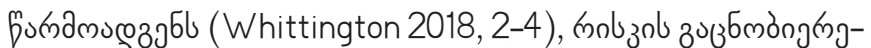

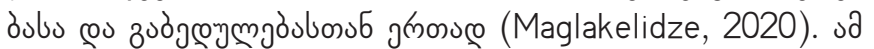

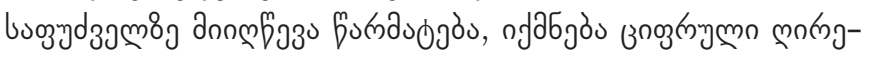

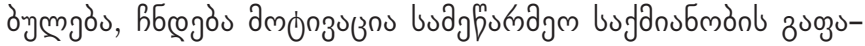

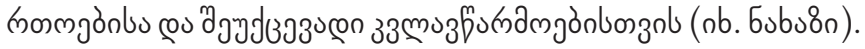

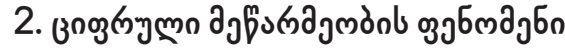

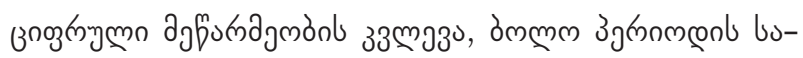
agzбngme modg

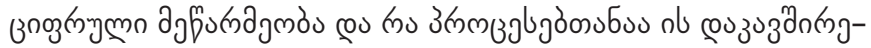

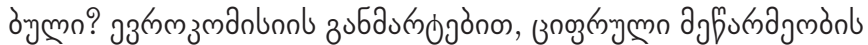

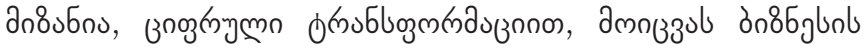

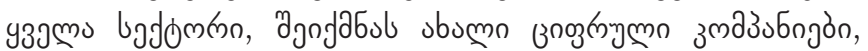

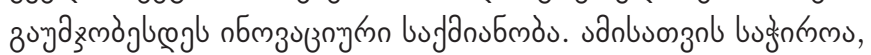

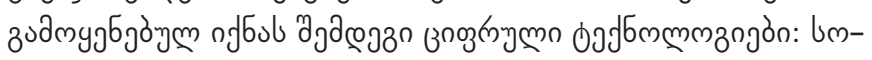

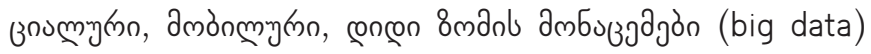
co cूñòmmzubn (cloud) zucoufysadjòn (European Commission, 2015: 14). змmаa

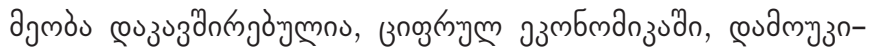

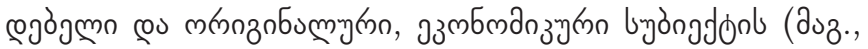

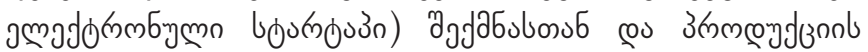

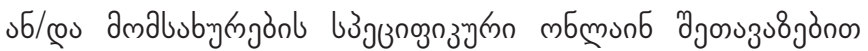

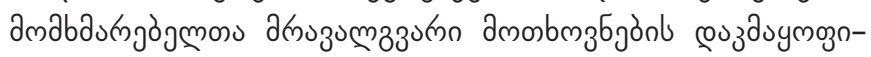

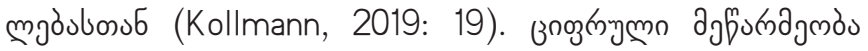

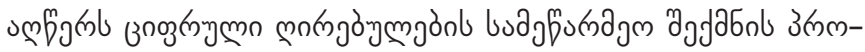

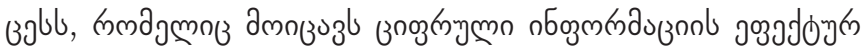

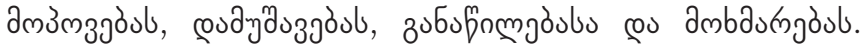

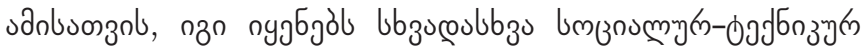

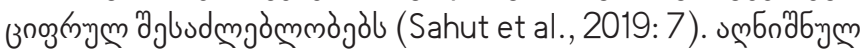

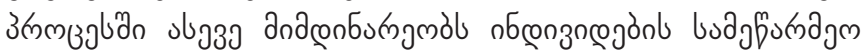

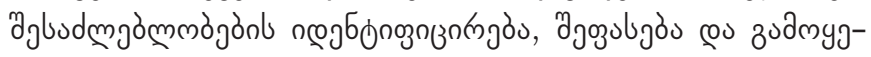

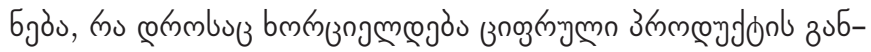

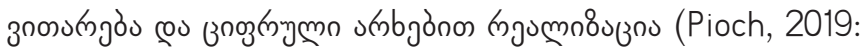

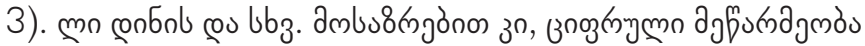

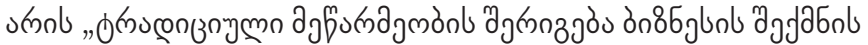

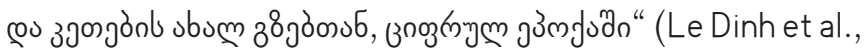
2018: 1).

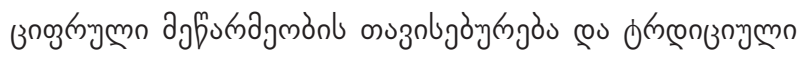

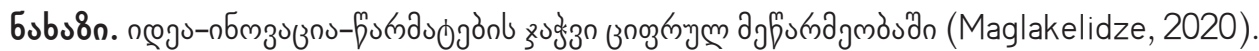

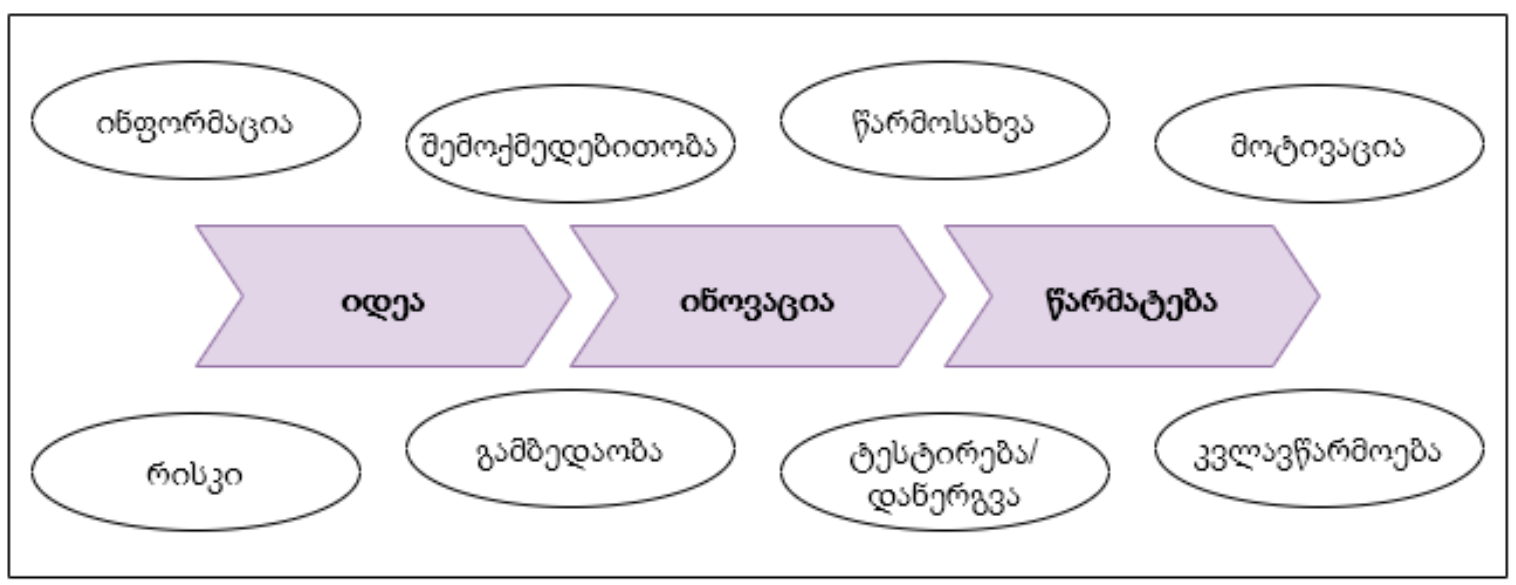




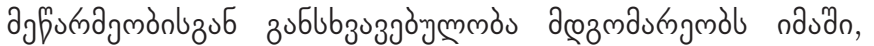

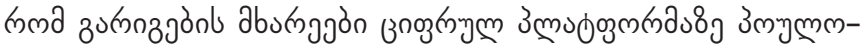

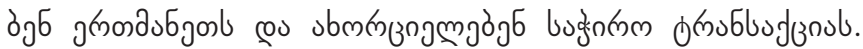

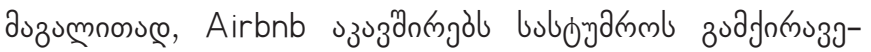

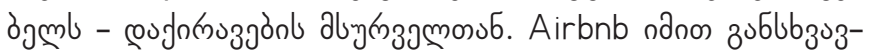

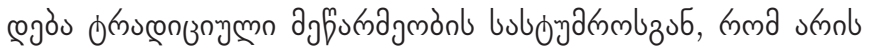

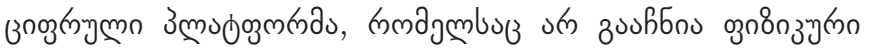

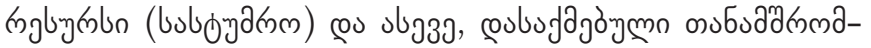

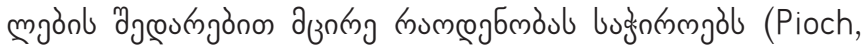

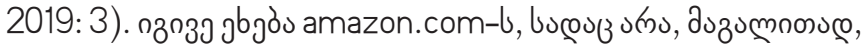

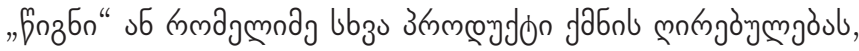

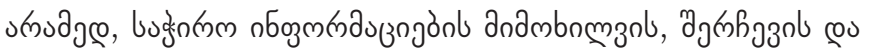

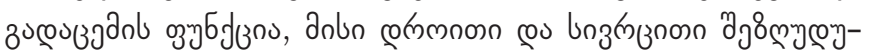

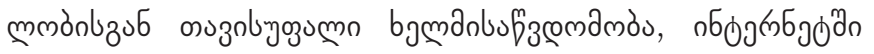

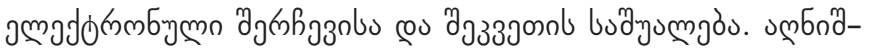

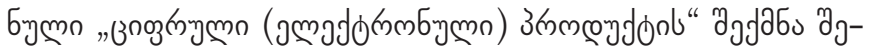

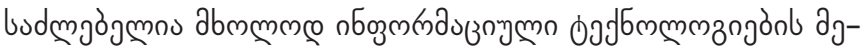
ə̆

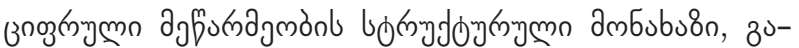

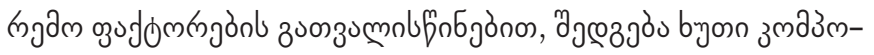

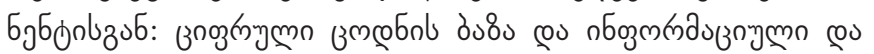

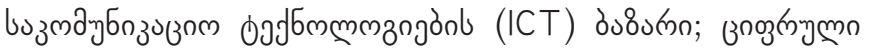

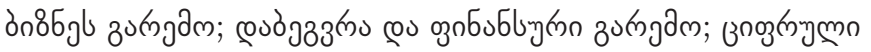

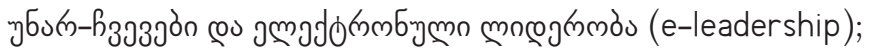

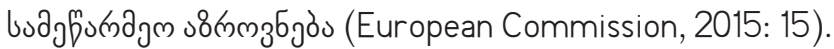

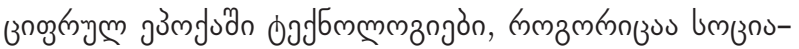

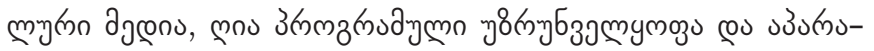

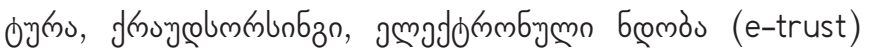

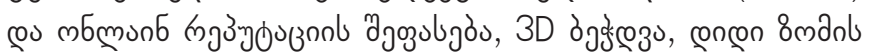

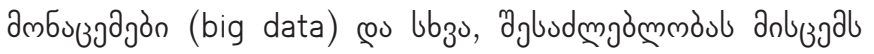

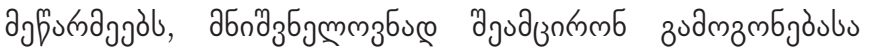

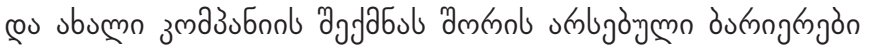

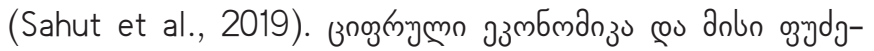

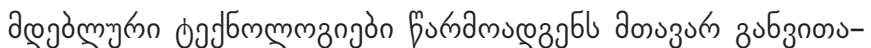

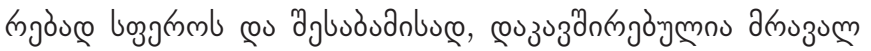

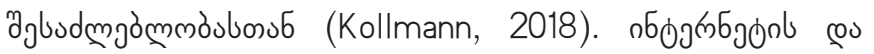

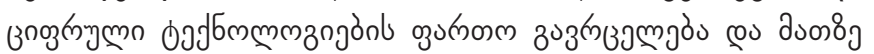

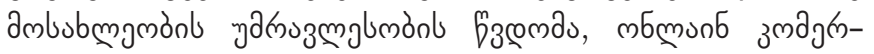

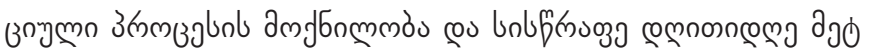

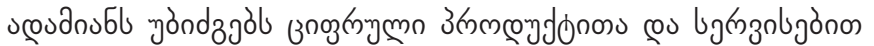

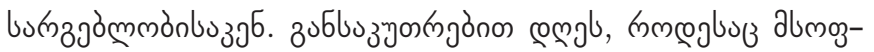

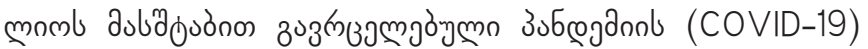

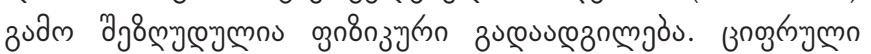

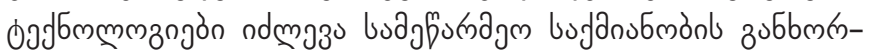

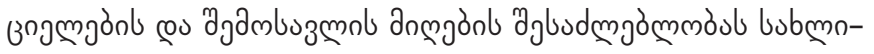

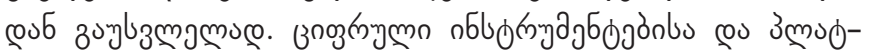

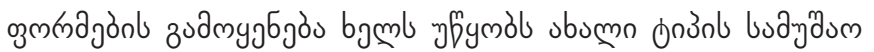
uçammgònb zuhgbul (Sahut et al., 2019).

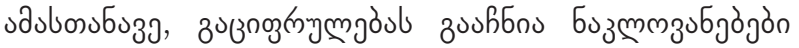

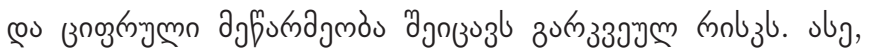

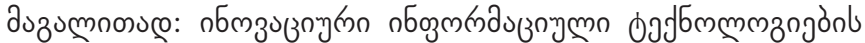

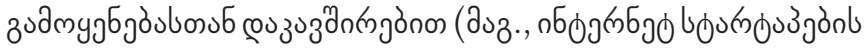

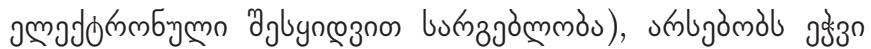

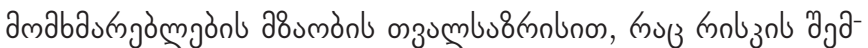

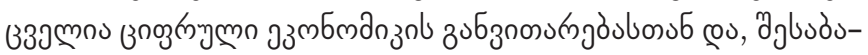

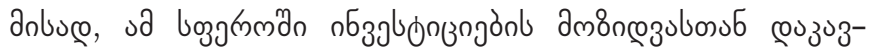

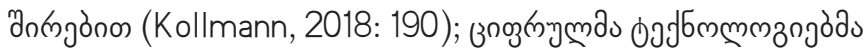

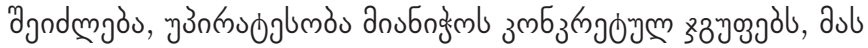

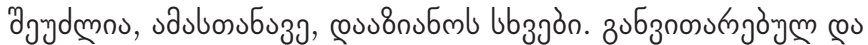

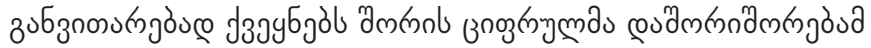

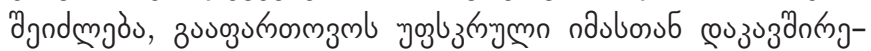

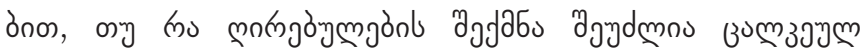

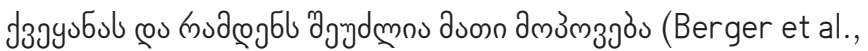

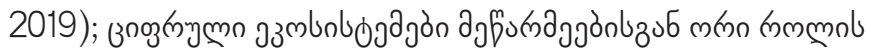

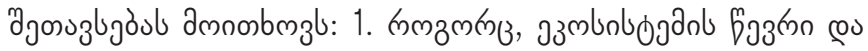

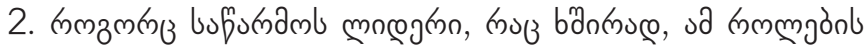

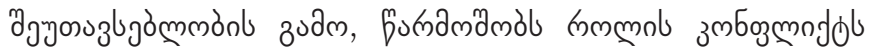
cou bofmglob domum combjl (Nambisan \& baron, 2019);

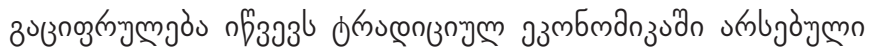

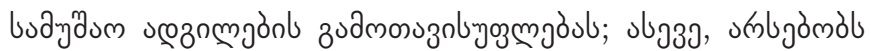

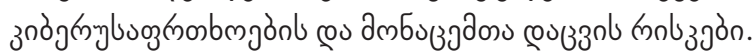

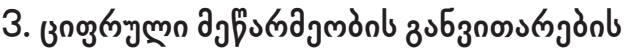

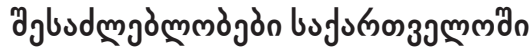

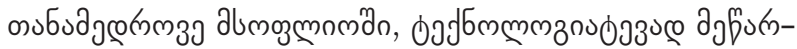

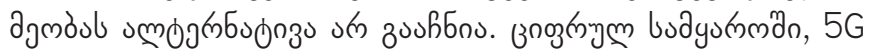

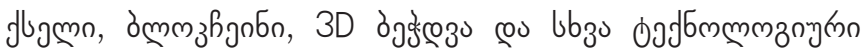

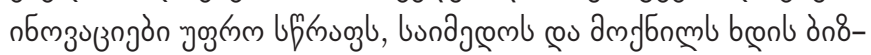

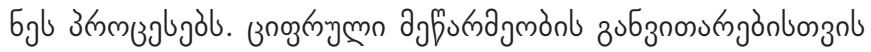

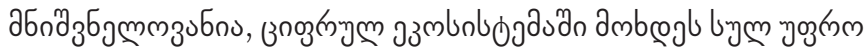

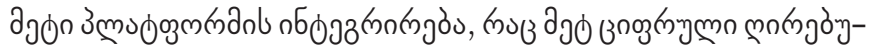

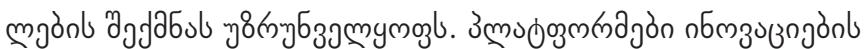

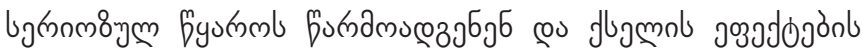

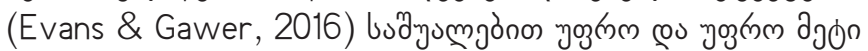

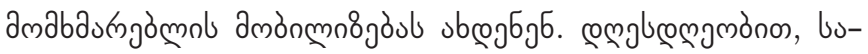

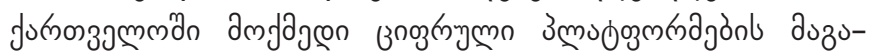

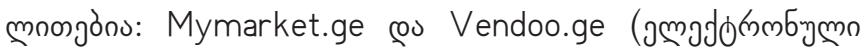

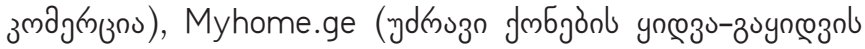

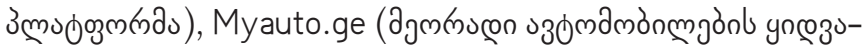

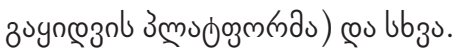

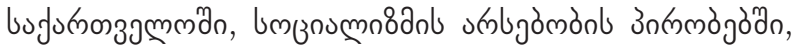

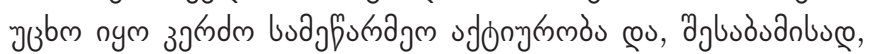

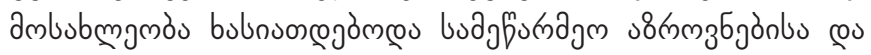

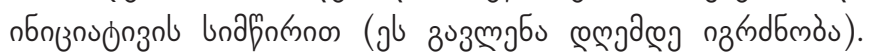

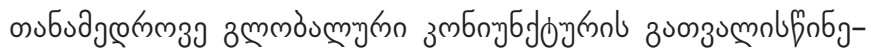

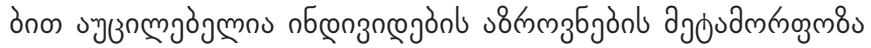

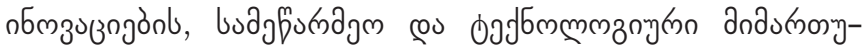

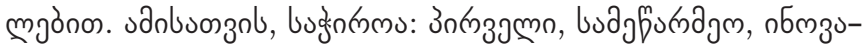

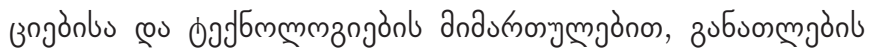




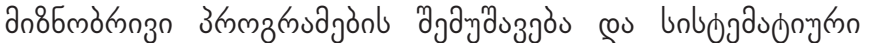

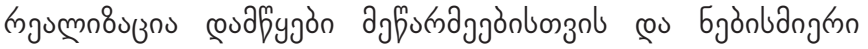

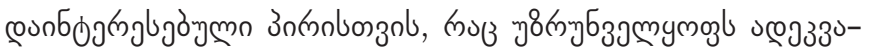

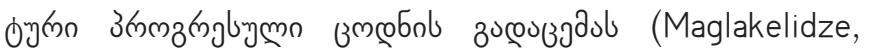

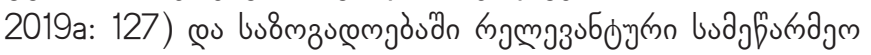

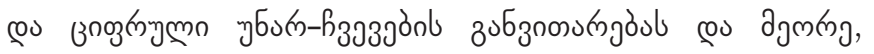

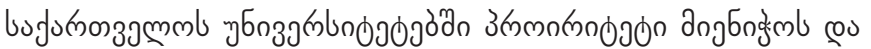

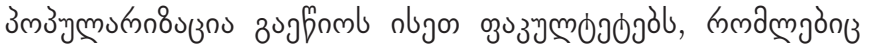

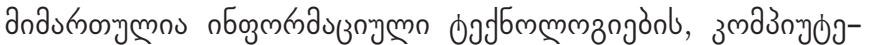

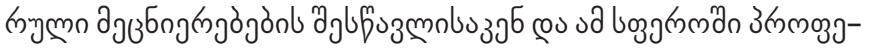

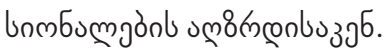

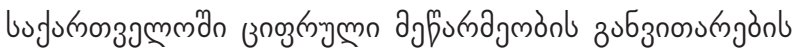

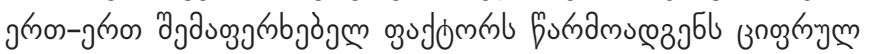

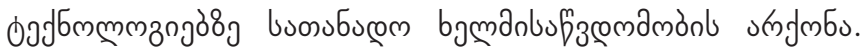

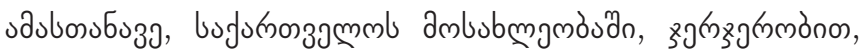

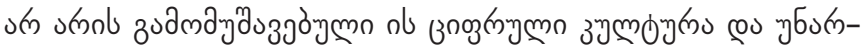

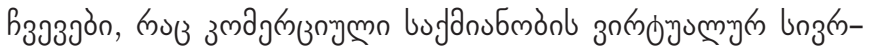

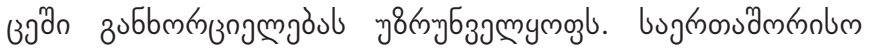

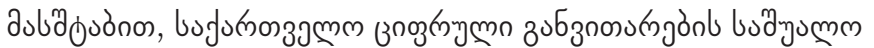

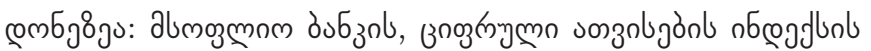

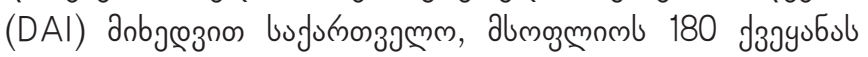

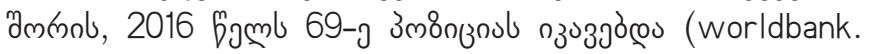
org); BBVA Research-nl उnogrnymn nбegflunb (DiGiX)

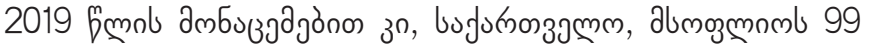

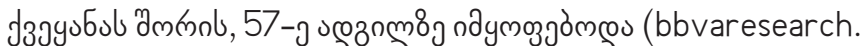
com). bufurnoszammbl boujnbonzol grnmзб

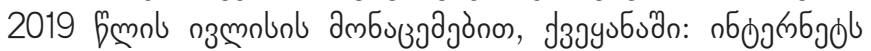

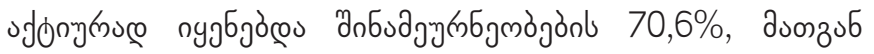

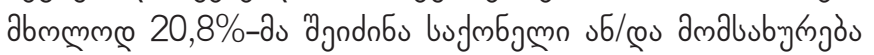

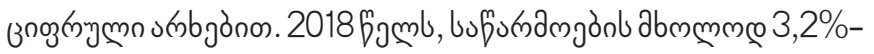

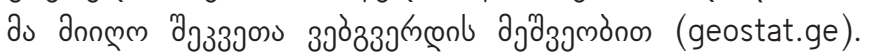

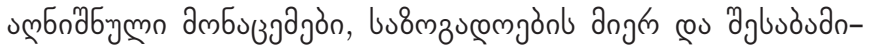

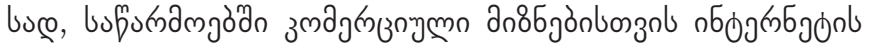

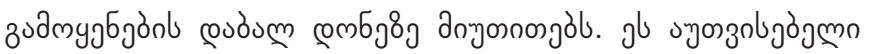

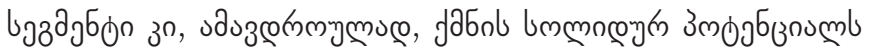

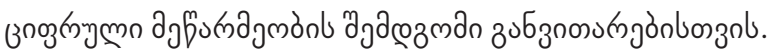

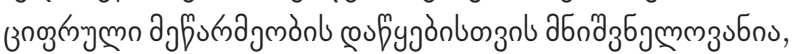

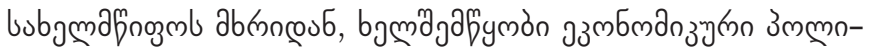

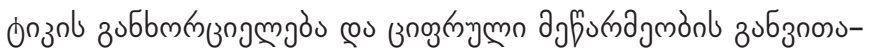

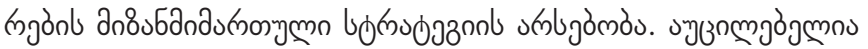

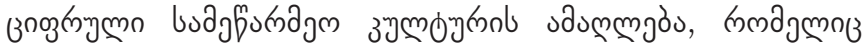

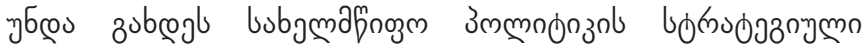

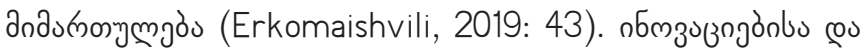

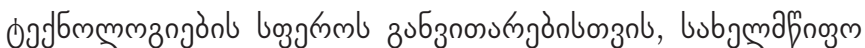

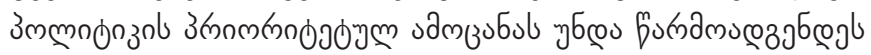

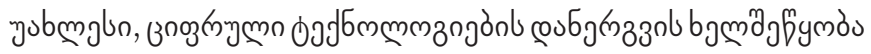
(Maglakelidze, 2019b: 175).

\section{cos335s}

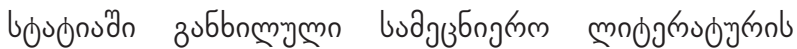

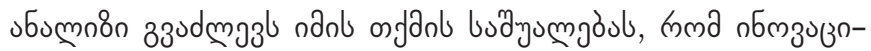

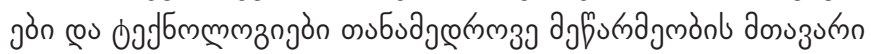

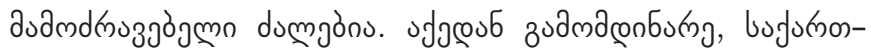

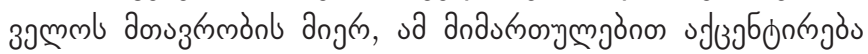

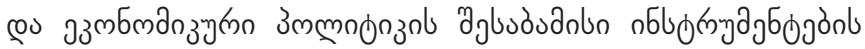

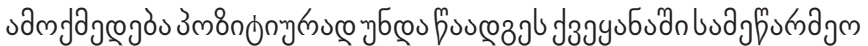

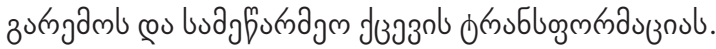

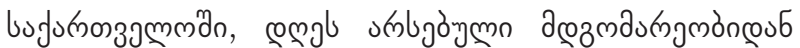

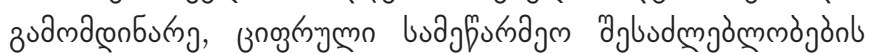

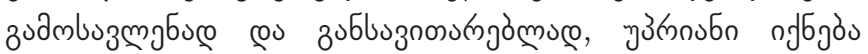

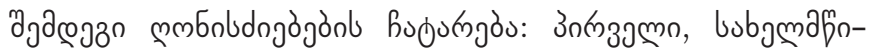

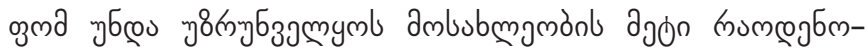

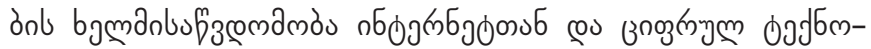

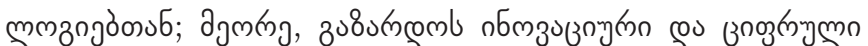

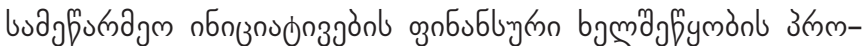

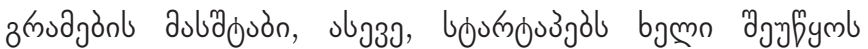

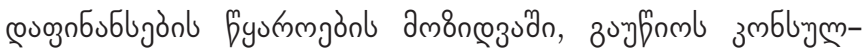

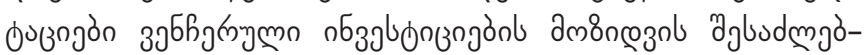

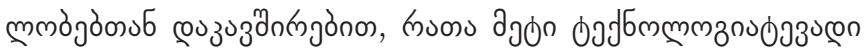

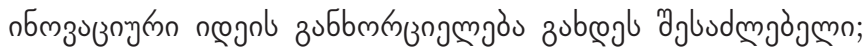

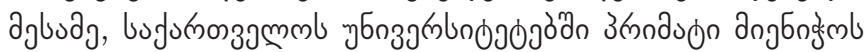

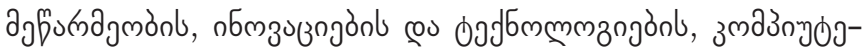

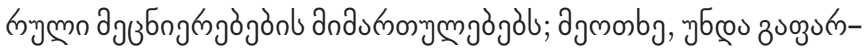
мmзৎgl

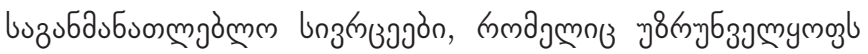

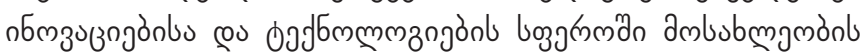

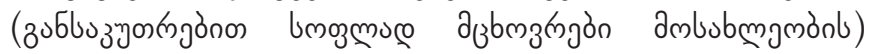
ojلб

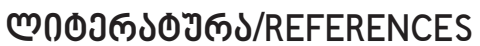

Bedianashvili, G. (2018) Culture as a Factor of Knowledge Economics with Paradigmatic Changes in Systemic Institutional Context. Journal Globalization and Business, N6, P.58-66 (In Georgian).

Bedianashvili, G. (2017) Formation of Knowledge Economy and Innovative Entrepreneurial Policy: Institutional Aspects. Journal Globalization and Business, N3, P.10-16 (In Georgian).

Erkomaishvili, G. (2019) Digital Economy - An Innovative Tool for Economic Growth. Journal Economics and Business, V.XI, N2, P.37-46 (In Georgian). 
Maglakelidze, A. (2020) Challenges and Perspectives of Digital Entrepreneurship in Georgia. Journal "Economics and Business, V.XII, N3 (In Georgian).

Maglakelidze, A. (2019a) Development of Small and Medium Entrepreneurship and Formation of a Middle Class in Georgia. Journal "Globalization and Business", N7, P.123-129 (In Georgian).

Maglakelidze, A. (2019b) For the Purpose of Forming National Innovation System in Georgia. Journal "Economics and Business", V.XI, N1, P.171-179 (In Georgian).

Berger, E. S. C., Briel, F., Davidsson, \& P., Kuckertz, A. (2019) Digital or not - The future of entrepreneurship and innovation. Journal of Business Research. DOI: 10.1016/j.jbusres.2019.12.020

European Commission (2015) Digital Entrepreneurship Scoreboard 2015. Brussels. https://ec.europa.eu/growth/content/ digital-entrepreneurship-scoreboard-2015-0_en

Evans, P. C. \& Gawer, A. (2016) The rise of the platform enterprise: a global survey. https://www.thecge.net/app/ uploads/2016/01/PDF-WEB-Platform-Survey_01_12.pdf

Kollmann, T. (2018) E-Entrepreneurship: Unternehmensgründung in der Net Economy. In Handbuch Entrepreneurship, Hrsg. G. Faltin, 181-199. Springer Gabler, Wiesbaden.

Kollmann, T. (2019) E-Entrepreneurship: Grundlagen der Unternehmensgründung in der digitalen Wirtschaft. 7. Auflage, Springer Gabler, Wiesbaden.

Le Dinh, T., Vu, M.C. \& Ayayi, A. (2018) Towards a living lab for promoting the digital entrepreneurship process. International Journal of Entrepreneurship, 22(1), 1-17.

Nambisan, S. \& Baron, R. A. (2019) On the costs of digital entrepreneurship: Role conflict, stress, and venture performance in digital platform-based ecosystems. Journal of Business Research. DOI: https://doi.org/10.1016/j.jbusres.2019.06.037

Nambisan, S. Wright, M. \& Feldman, M. (2019) The digital transformation of innovation and entrepreneurship: Progress, challenges and key themes. Research Policy, 48(8). DOI: https://doi.org/10.1016/j.respol.2019.03.018

Piegeler, M. (2015) Entrepreneurship und Innovation: Stand der Forschung und politische Handlungsempfehlungen, IWAnalysen, No. 103, Institut der deutschen Wirtschaft (IW), Köln.

Pioch, S. (2019) Digital Entrepreneurship: ein Praxisleitfaden für die Entwicklung eines digitalen Produkts von der Idee bis zur Markteinführung. Springer Gabler, Wiesbaden.

Runge, W. (2014) Technology Entrepreneurship: a Treatise on Entrepreneurs and Entrepreneurship for and in Technology Ventures. Volume 1, KIT Scientific Publishing, Karlsruhe.

Sahut, J., Iandoli, L. \& Teulon, F. (2019) The age of digital entrepreneurship. Journal Small Business Economics. DOI: https:// doi.org/10.1007/s11187-019-00260-8

Schumpeter, J. (1931) Theorie der wirtschaftlichen Entwicklung: eine Untersuchung über Unternehmergewinn, Kapital, Kredit, Zins und den Konjunkturzyklus. Dritte Auflage. Verlag von Duncker \& Humblot, München und Leipzig.

Whittington, D. (2018) Digital Innovation and Entrepreneurship. Cambridge University Press, Cambridge.

https://www.geostat.ge/ka/modules/categories/104/sainformatsio-da-sakomunikatsio-teknologiebi /Last view 19 May, 2020/

https://www.bbvaresearch.com/en/publicaciones/digix-2019-tracking-digital-evolution//Last view 19 May, 2020/

https://www.worldbank.org/en/publication/wdr2016/Digital-Adoption-Index/Last view 19 May, 2020/ 